\title{
Confiabilidad, validez de criterio y discriminante del Montreal Cognitive Assessment (MoCA) test, en un grupo de adultos de Bogotá
}

\author{
Reliability, criterion and discriminant validity of the \\ Montreal Cognitive Assessment Test (MoCA) in a \\ group of adults from Bogotá
}

\author{
Olga lucía Pedraza, Ana María Salazar, Fabio Alexander Sierra, \\ David Soler, July Castro, Pablo Castillo, Angélica Hernández, \\ César Piñeros • Bogotá, D.C. (Colombia)
}

\section{Resumen}

Introducción: el MoCA-test es un instrumento breve de tamizaje, sensible y específico, utilizado para la detección del deterioro cognitivo leve (DCL) y la demencia, con puntos de corte que varían según la población estudiada.

Objetivo: evaluar la confiabilidad y validez discriminante del MoCA-test, en un grupo de adultos de Bogotá, con diferentes escolaridades.

Material y Métodos: se aplicó el MoCA-test y el MMSE, a 1174 adultos mayores de 50 años de diferentes localidades de Bogotá. Los sujetos con MoCA-test < 26 y MMSE <24, fueron citados a una segunda evaluación aplicándoseles un protocolo amplio y fueron analizados por consenso, para determinar normalidad o deterioro cognitivo. Se determinó la consistencia interna (con coeficiente alpha de Cronbach), confiabilidad test-retest (con coeficiente de Lin), la validez de criterio (con coeficiente de Spearman) y validez discriminante (por medio de curvas ROC) del MoCA-Test.

Resultados: la consistencia interna (alfa-Cronbach=0.851) y la confiabilidad test-retest (Lin=0.62) fueron aceptables. La validez de criterio respecto al MMSE, fue moderada ( $r=0.65)$. El MoCA mostró capacidad para discriminar entre diferentes grupos diagnósticos y sociodemográficos. El área bajo la curva fue 0.76 para DCL y 0,81 para demencia; el punto de corte para discriminar entre normalidad y DCL en el grupo en general fue 20/21 y entre DCL y demencia 17/18. Estos puntos variaron con el grado de escolaridad.

Conclusión: el MoCA-test puede ser el instrumento de tamización en atención primaria, para detectar deterioro cognitivo en nuestra población adulta, considerando los puntos de corte propuestos según la escolaridad. (Acta Med Colomb 2016; 40: 221-228).

Palabras clave: MoCA-test, MMSE, validación en español, puntos de corte, población adulta.

\footnotetext{
Abstract

Introduction: the MoCA-Test is a brief, sensitive and specific screening tool used to detect mild cognitive impairment (MCI) and dementia, with cut-off points that vary according to the population studied.

Objective: to evaluate the reliability and discriminant validity of the MoCA-test, in a group of adults from Bogotá, with different levels of schooling.

Material and Methods: the MoCA-test and the MMSE were applied to 1174 adults over 50 years old from different locations in Bogotá. Subjects with MoCA-test <26 and MMSE <24, were referred to a second evaluation by applying a broad protocol and were analyzed by consensus, to determine normality or cognitive impairment. Internal consistency (with Cronbach's alpha coefficient), test-retest reliability (with Lin coefficient), criterion validity (with Spearman's coefficient) and discriminant validity (using ROC curves) of the MoCA-Test were determined.
}

Dra. Olga Lucía Pedraza: Grupo Interdisciplinario de Memoria, Hospital Infantil Universitario de San José (HIUSJ). Grupo de Neurociencias Fundación Universitaria de Ciencias de la Salud (FUCS); Dra. Ana María Salazar: Grupo Interdisciplinario de Memoria, Hospital Infantil Universitario de San José (HIUSJ). Grupo Procesos Cognoscitivas y de la Emoción, Facultad de Psicología Universidad El Bosque; Dr. Fabio Alexander Sierra: Epidemiología y Bioestadística. División de Investigación de la FUCS; Dr. David Soler y Dra. July Castro: Estudiantes Semillero de Neurociencias, Facultad de Medicina FUCS; Pablo Castillo y Angélica Hernández: Estudiantes Facultad de Psicología, Universidad El Bosque; Dr. César Piñeros: Epidemiología y Bioestadística, División de Investigación de la FUCS. Bogotá, D.C. (Colombia).

Correspondencia: Dra. Olga Lucía Pedraza. Bogotá, D.C. (Colombia).

E-mail: opedraza@hospitalinfantildesanjose. org.co

Recibido: 20/X/2015 Aceptado: 27/X/2016 